\title{
FOLL PAPERS
}

\section{Central Venous Catheter (CVC) related infections: a local retrospective study}

\author{
Manuela Fresu', Agostina Ronca', Carla Pruzzo', Simona Roveta ${ }^{3}$ \\ 'Struttura Semplice Dipartimentale di Microbiologia - Azienda Ospedaliera Santa Corona, \\ Via XXV Aprile 38, I 7027 Pietra Ligure (SV) \\ ${ }^{2}$ Università di Genova, Dipartimento di Biologia, Corso Europa 26, I6I 32 Genova \\ ${ }^{3}$ Università di Genova, DISCAT - Sezione di Microbiologia, Largo R. Benzi 10, I6I32 Genova
}

\section{Central Venous Catheter (CVC) related infections: a local retrospective study}

Key word: Central Venous Catheter; Catheter-related bloodstream, Infection; Quantitative culture

\section{SUMMARY}

Background. Central venous catheter (CVC) related infection is associated with significant increases in morbidity, mortality, and health care cost. This local surveillance study was carry out to monitor the frequency of occurrence of CVC-related blood stream infections.

Materials and methods. During the period January - December 2005, 226 CVC specimens were analyzed (quantitative method) and microrganism identification from positive samples was performed by Vitek II. In 53 patients it was possible to compare quantitative results with those obtained from blood cultures.

Results. Positive CVC samples were 125 (55\%) and 130 microrganisms were isolated: 109 Gram-positives (84\%), 4 Gram-negatives (3\%), and 17 mycetes (13\%).

Among pathogens collected simultaneously from CVC and blood samples, the most frequently isolated were Staphylococcus spp. (30\% coagulase-negative staphylococci and 20\%. S. aureus) and Candida spp. (45\%). In the group of patients that presented positive CVC and negative blood samples the most frequently recovered microrganisms were staphylococci. Many isolates (33\%) were polymicrobial.

Conclusions. Catheter-related infections occurred in those patients who presented the same pathogen in both CVC and blood cultures. These infections were principally caused by staphylococci and Candida spp. On the contrary, a possible CVC contamination could be suspected when positive CVC and negative blood cultures were found.

\section{INTRODUZIONE}

La cateterizzazione è una procedure invasiva che può favorire l'ingresso di microrganismi nel sangue e rappresenta, pertanto, un fattore di rischio per l'insorgenza di batteriemie e sepsi. Le infezioni catetere-correlate costituiscono una significativa causa di incremento sia della morbilità e della mortalità che dei costi e della durata dell'ospedalizzazione. L'aumento dell'incidenza delle infezioni correlate a catetere venoso centrale $(\mathrm{CVC})$ può dipendere da diversi fattori come il numero crescente di soggetti affetti da patologie per cui vi è l'indicazione al ricorso al CVC e il miglioramento della sensibilità delle indagini microbiologiche $(7-8,11)$.

Un quadro settico può essere attribuito ad un'infezione da catetere quando in presenza di segni clinici sistemici non vi siano apparentemente altri focolai di possibile infezione ad eccezione del catetere medesimo. Esistono varie modalità di rilevare un'infezione ematica correlata al catetere e tra queste è compresa quella che tiene conto dell'isolamento del medesimo microorganismo sia dal sangue periferico (emocoltura) che dal catetere. Le colture semi-quantitative o quantitative della punta del catetere, poiché presentano una maggiore specificità rispetto a quelle qualitative, sono le metodologie più frequentemente utilizzate per questo tipo di indagine diagnostica $(4-5,9)$. I principali agenti eziologici delle infezioni CVCcorrelate sono i cocchi Gram-positivi che costituiscono circa i due terzi degli isolati, con prevalenza relativa degli stafilococchi coagulasi-negativi (SCN). Non trascurabile la presenza dei microrganismi Gram-negativi (Enterobacteriaceae e Pseudomonas aeruginosa) e dei miceti (Candida 
albicans e Candida spp.) $(2,9)$.

In questo studio epidemiologico locale è stata condotta un'indagine retrospettiva analizzando i risultati di tutte le colture eseguite su CVC nel nostro laboratorio in un periodo di tempo di un anno. È stato, quindi, valutato quanti pazienti con CVC positivo presentavano lo stesso germe anche dall'isolamento proveniente da emocoltura (diagnosi di sepsi catetere correlata).

\section{MATERIALI E METODI}

Nel periodo gennaio-dicembre 2005, presso la Struttura Semplice Dipartimentale di Microbiologia dell'Azienda Ospedaliera Santa Corona, sono stati analizzati $226 \mathrm{CVC}$ provenienti da pazienti ospedalizzati (reparti di Chirurgia, Rieducazione Funzionale, Medicina Generale e Rianimazione). In parallelo sono state valutate anche le eventuali emocolture effettuate sui pazienti in cui l'indagine microbiologica relativa al CVC aveva dato esito positivo.

L'espianto del catetere e la preparazione per l'analisi microbiologica sono stati effettuati facendo particolare attenzione ad evitare possibili contaminazioni da contatto con superfici non sterili. La procedura prevedeva, previa disinfezione della cute peri-catetere, l'estrazione del CVC utilizzando una pinza sterile, il taglio della punta (segmento di lunghezza non oltre i $4 \mathrm{~cm}$ ) e l'introduzione in un contenitore, anch'esso sterile. Il campione così prelevato giungeva al nostro laboratorio nel più breve tempo possibile (non oltre $\mathrm{i}$ 15 minuti). Ciascun campione è stato analizzato tramite coltura quantitativa: dapprima si procedeva all'aggiunta di Tryptic Soy Broth (TSB) e all'agitazione con vortex per 30 secondi, quindi alla semina di diluizioni seriali su piastre di Columbia agar addizionato con sangue di montone al $5 \%$ e, infine, all'incubazione in termostato per $18-24$ ore. Sono stati considerati positivi i campioni che presentavano una crescita di almeno $10^{2} \mathrm{CFU} / \mathrm{ml}(1,3,9)$.

Per ciascuna emocoltura venivano inviati al laboratorio 2 flaconi, uno per aerobi e uno per anaerobi, ciascuno inoculato al momento del prelievo con $5-10 \mathrm{ml}$ di sangue. La positività dei campioni è stata valutata con il sistema automatico BACT/ALERT $^{\circledR}$ 3D (bioMérieux Italia S.p.A.) che provvedeva all'incubazione $\left(\mathrm{a} 35^{\circ} \mathrm{C}\right)$ e alla visualizzazione automatizzata della curva di crescita dei microrganismi. Dai flaconi segnalati positivi è stato allestito un vetrino per la colorazione di Gram (10) e, parallelamente, si è proceduto alla semina su terreni idonei. I campioni risultati positivi sono stati seminati su 2 piastre di Columbia agar sangue 5\%, Mac-Conkey e Chapman (bioMérieux Italia S.p.A.), per ciascun tipo di terreno una piastra è stata incubata in aerobiosi ed una in anaerobiosi. È stata seminata anche 1 piastra di agar-cioccolato e incubata in anaerobiosi. Per l'identificazione dei germi è stato impiegato il metodo automatizzato Vitek II (bioMérieux Italia S.p.A).

\section{RISULTATI}

Sono risultati positivi all'esame colturale 125 CVC su un totale di 226 , pari al 55\% dei campioni analizzati. Il maggior numero di isolati proveniva dai reparti di Chirurgia $(33.8 \%)$ e dalla Rianimazione (31.5\%).

La tabella 1 riporta i 130 microrganismi isolati (5 isolamenti erano polimicrobici) suddivisi per reparto di provenienza. In particolare sono stati rinvenuti 109 Gram - positivi (48 Staphylococcus epidermidis, 19 Staphylococcus haemolyticus, 9 Staphylococcus aureus, 23 stafilococchi coagulasi negativi (SCN), 9 Enterococcus faecalis, 1 Bacillus spp.) 4 Gram-negativi (2 Pseudomonas aeruginosa, 1 Stenotrophomonas maltophilia, 1 Escherichia coli) e 17 miceti del genere Candida (5 C. albicans, 3 C. tropicalis, 2 C. glabrata, 6 C. parapsilosis, 1 C. famata) (figura I).

Solamente per 53 pazienti su 226 sono state richieste sia la coltura della punta del CVC che l'emocoltura, nei rimanenti casi l'emocoltura non è stata eseguita. Per 38 pazienti (i rimanenti 15 presentavano entrambe le colture negative) è stato possibile confrontare i risultati della coltura relativa al CVC con quelli dell'emocoltura: 20 $(52.6 \%)$ presentavano positività per lo stesso patogeno sia nell'emocoltura che nel CVC, mentre $18(47.4 \%)$ mostravano crescita batterica nel $\mathrm{CVC}$ con emocolture negative. In particolare, i 20 microrganismi isolati (tutti gli isolamenti erano monomicrobici) parallelamente da CVC ed emocoltura sono stati 10 Gram-positivi (4 S. aureus, 3 $S$. epidermidis, $2 S$. haemolyticus, 1 Staphylococcus hominis), 1 Gram-negativo ( $P$. aeruginosa) e 9 miceti, tutti appartenenti al genere Candida (2 C. albicans, 2 C. tropicalis, $3 C$. parapsilosis, 1 C. glabrata e 1 C. famata) (figura II). I risultati sono riportati in dettaglio nella tabella 2A. Nel gruppo caratterizzato da CVC positivo-emocolture negative (tabella $2 \mathrm{~B}$ ) sono stati spesso evidenziati isolamenti polimicrobici $(33 \%)$ e la quasi totalità dei microrganismi isolati apparteneva al genere Staphylococcus.

\section{CONCLUSIONI}

Si può presumibilmente parlare di infezioni catetere correlate quando lo stesso patogeno viene isolato sia dal CVC che dal sangue $(5,9)$. Se confrontiamo le percentuali degli isolati da $\mathrm{CVC}$ con quelle dei microrganismi isolati contemporanea- 
Tabella I. Microrganismi isolati da CVC in relazione ai reparti di provenienza

\begin{tabular}{|c|c|c|c|c|c|}
\hline Microrganismo & Chirurgia $^{(a)}$ & $\begin{array}{l}\text { Rieducazione } \\
\text { Funzionale }\end{array}$ & $\begin{array}{l}\text { Medicina } \\
\text { Generale }\end{array}$ & Rianimazione & Totale \\
\hline S. epidermidis & 14 & 7 & 7 & 20 & 48 \\
\hline S. haemolyticus & 9 & 3 & $\mathrm{I}$ & 6 & 19 \\
\hline S. aureus & 4 & - & 3 & 2 & 9 \\
\hline $\mathrm{SCN}^{(\mathrm{b})}$ & 6 & 3 & 8 & 6 & 23 \\
\hline E. faecalis & 3 & 2 & $\mathrm{I}$ & 3 & 9 \\
\hline Bacillus spp. & I & - & - & - & $\mathrm{I}$ \\
\hline P. aeruginosa & - & - & - & 2 & 2 \\
\hline S. maltophilia & - & I & - & - & I \\
\hline$\overline{\text { E. coli }}$ & I & - & - & - & I \\
\hline C. albicans & $\mathrm{I}$ & $\mathrm{I}$ & 3 & - & 5 \\
\hline C. tropicalis & $\mathrm{I}$ & - & $\mathrm{I}$ & I & 3 \\
\hline C. glabrata & I & - & - & I & 2 \\
\hline C. parapsilosis & 3 & I & 2 & - & 6 \\
\hline C. famata & - & - & I & - & 1 \\
\hline Totale & 44 & 18 & 27 & 41 & 130 \\
\hline
\end{tabular}

(a) Chirurgia Generale, Chirurgia Ortopedica, Chirurgia Plastica, Otorinolaringoiatria.

(b) 17 Staphylococcus hominis, 6 Staphylococcus simulans.

Tabella 2. Microrganismi isolati parallelamente da CVC ed emocoltura (A) e con CVC positivo ed emocoltura negativa (B) suddivisi per reparti di provenienza

\begin{tabular}{|c|c|c|c|c|c|}
\hline $\mathbf{A}$ & & & & & \\
\hline Microrganismo & Chirurgia $^{(a)}$ & $\begin{array}{c}\text { Rieducazione } \\
\text { Funzionale }\end{array}$ & $\begin{array}{l}\text { Medicina } \\
\text { Generale }\end{array}$ & Rianimazione & Totale \\
\hline S. epidermidis & - & I & - & 2 & 3 \\
\hline S. haemolyticus & $\mathrm{I}$ & - & - & I & 2 \\
\hline S. aureus & 2 & - & $\mathrm{I}$ & $\mathrm{I}$ & 4 \\
\hline S. hominis & $\mathrm{I}$ & - & - & - & I \\
\hline C. albicans & - & - & 2 & - & 2 \\
\hline C. tropicalis & $\mathrm{I}$ & - & - & $\mathrm{I}$ & 2 \\
\hline C. glabrata & I & - & - & - & I \\
\hline C. parapsilosis & I & I & 1 & - & 3 \\
\hline C. famata & - & - & $\mathrm{I}$ & - & I \\
\hline P. aeruginosa & - & - & - & I & I \\
\hline Totale & 7 & 2 & 5 & 6 & 20 \\
\hline
\end{tabular}

(a) Chirurgia Generale, Chirurgia Ortopedica, Chirurgia Plastica, Otorinolaringoiatria.

\begin{tabular}{|c|c|c|c|c|c|}
\hline \multirow{2}{*}{$\frac{\text { B }}{\text { Microrganismo }}$} & \multicolumn{4}{|c|}{ Reparti } & \multirow[b]{2}{*}{ Totale } \\
\hline & Chirurgia $^{\text {(a) }}$ & $\begin{array}{l}\text { Rieducazione } \\
\text { Funzionale }\end{array}$ & $\begin{array}{l}\text { Medicina } \\
\text { Generale }\end{array}$ & Rianimazione & \\
\hline S. epidermidis & I & I & I & 7 & 10 \\
\hline S. haemolyticus & 4 & $\mathrm{I}$ & - & $\mathrm{I}$ & 6 \\
\hline S. aureus & - & - & I & & I \\
\hline $\mathrm{SCN}^{(\mathrm{b})}$ & $\mathrm{I}$ & - & - & 2 & 3 \\
\hline E. faecalis & - & - & - & $\mathrm{I}$ & $\mathrm{I}$ \\
\hline C. albicans & - & - & $\mathrm{I}$ & - & $\mathrm{I}$ \\
\hline C. parapsilosis. & $\mathrm{I}$ & - & - & - & $\mathrm{I}$ \\
\hline Bacillus spp. & I & - & - & - & I \\
\hline Totale & 8 & 2 & 3 & I I & 24 \\
\hline
\end{tabular}

(a) Chirurgia Generale, Chirurgia Ortopedica, Chirurgia Plastica, Otorinolaringoiatria.

(b) 2 Staphylococcus hominis, I Staphylococcus simulans

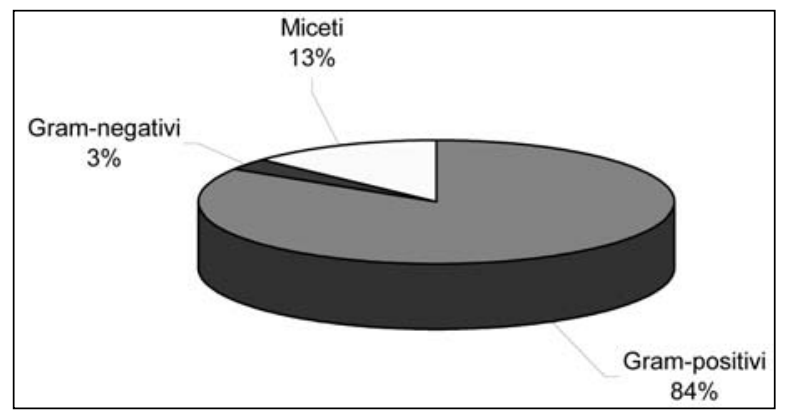

Figura I. Distribuzione dei 130 microrganismi isolati da CVC

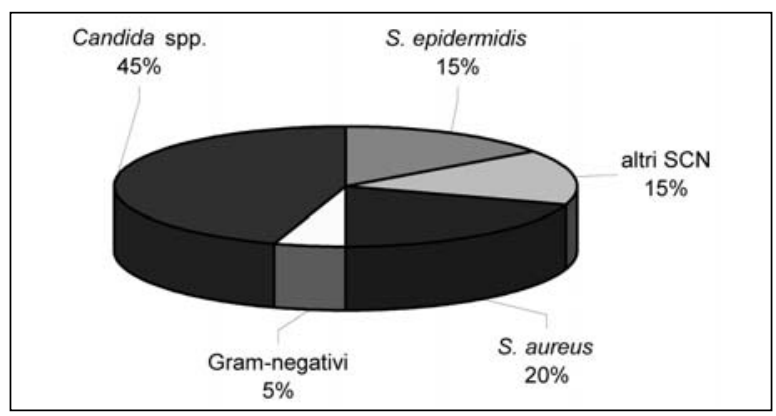

Figura II. Distribuzione dei 20 microrganismi isolati parallelamente da CVC ed emocoltura 
mente da CVC e da emocoltura possiamo trovare alcune importanti differenze, già precedentemente segnalate in letteratura (6). I Gram-positivi (appartenenti per la quasi totalità al genere Staphylococcus) rappresentano 1' $84 \%$ degli isolati da CVC, ma la percentuale scende al $50 \%$ quando si considerano solo gli isolati per cui vi è il medesimo riscontro anche nell'emocoltura. Parallelamente, i miceti del genere Candida che rappresentano il 13\% degli isolati da CVC, assumono un maggior rilievo (45\%) quando andiamo ad analizzare solamente quei casi in cui è stato possibile isolare il germe anche dal sangue. Per quanto riguarda i Gram-negativi, invece, la percentuale resta sempre al di sotto del 5\%. I reparti in cui sono state evidenziate la maggior parte di infezioni CVC correlate sono stati quelli di Chirurgia (35\%) e Rianimazione (30\%).

Gli isolati che presentavano CVC positivo ma emocolture negative erano rappresentati per circa l' $80 \%$ da SCN. La positività di questi campioni può essere legata alla colonizzazione del catetere da parte di questi microrganismi che si trovano comunemente sulla cute. Molti isolati, inoltre, erano di natura polimicrobica: questo dato suggerisce che si siano verificate contaminazioni esogene causate da una non corretta pratica di prelievo del campione.

Sugli 87 casi di CVC positivi con emocolture assenti non è stato possibile fare alcuna valutazione su una possibile sepsi catetere correlata. Questo elevato numero di richieste di coltura della punta di CVC non accompagnata da emocoltura fa riflettere su quanto sia ancora poco utilizzato un iter diagnostico corretto (invio contemporaneo di emocoltura periferica e punta di CVC).

\section{BIBLIOGRAFIA}

1. Bouza E, Burillo A, Munoz P. Catheter-related infections: diagnosis and intravascular treatment. Clin Microbiol Infect 2002; 8: 265-74.

2. Bouza E, San Juan R, Munoz P, Pascau J, Voss A, Desco M, Cooperative Group of the European Study Group on Nosocomial Infections (ESGNI). A European perspective on intravascular catheter-related infections: report on the microbiology workload, aetiology and antimicrobial susceptibility (ESGNI005 Study). Clin Microbiol Infect 2004; 10: 838-42.

3. Cleri DJ, Corrado ML, Seligman SJ. Quantitative culture of intravenous catheters and other intravascular inserts. J Infect Dis 1980; 141: 781-6.

4. Crump JA, Collignon PJ. Intravascular catheter-associated infections. Eur $\mathrm{J}$ Clin Microbiol Infect Dis 2000; 19: 1-8.

5. Hanna R, Raad II. Diagnosis of catheter-related bloodstream infection. Curr Infect Dis Rep 2005; 7: 413-9.

6. Lin C, Lin MT, Hsieh DY, et al. Microbiology difference between colonized catheters and catheter-related bloodstream infections. Hepatogastroenterology 2003; 50: 1821-4.

7. Lorente L, Henry C, Martin MM, Jimenez A, Mora ML. Central venous catheter-related infection in a prospective and observational study of 2595 catheters. Crit Care 2005; 9: R631-5.

8. Lorente L, Villegas J, Martin MM, Jimenez A, Mora ML. Catheter-related infection in critically ill patients. Intensive Care Med 2004; 30: 1681-4.

9. Mermel LA, Farr BM, Sherertz RJ, et al. Guidelines for the management of intravascular catheter-related infections. Clin Infect Dis 2001; 32: 1249-72.

10. Murray PR, Baron EJ, Jorgensen JH, Pfaller MA, Yolken RH. Manual of clinical microbiology. 8th Edition. American Society of Microbiology Press. Washington DC, 2003.

11. Polderman KH, Girbes AR. Central venous catheter use. Part 2: infectious complications. Intensive Care Med 2002; 28: 18-28. 